\title{
Risco de Crédito e Alocação Ótima para uma Carteira de Debêntures
}

\author{
- ANdré CADIME de GodóI* - JOE AKIRA YOSHINO ** \\ - ROgÉRIO de DEUS OliveirA***
}

\begin{abstract}
RESUMO
A debênture vem se tornando um instrumento de captação cada vez mais importante para empresas não financeiras no mercado brasileiro e uma alternativa às elevadas taxas de juros cobradas pelos bancos comerciais em uma operação de financiamento. Um aspecto-chave para o desenvolvimento do mercado secundário deste instrumento é o correto tratamento do risco de crédito, que ocorre quando o emissor não cumpre suas obrigações contratuais. Este trabalho propõe e testa uma metodologia que determina a magnitude deste risco para uma carteira de debêntures de empresas emissoras brasileiras. A abordagem utilizada baseia-se no Modelo de Merton (1974) para bônus corporativos, que utiliza as fórmulas de Black-Scholes para o cálculo do preço de opções. Também são utilizadas técnicas de otimização para a determinação do risco da carteira. Adotando um modelo simples e de baixo custo computacional, chegamos a uma medida de risco mais conservadora do que a obtida com o tradicional modelo VaR (value at risk). Além disso, apresentamos uma metodologia para a obtenção da composição ótima da carteira de debêntures.
\end{abstract}

\section{Palavras-Chave}

risco de crédito, títulos corporativos, perda máxima, Modelo de Merton

\begin{abstract}
The debenture (corporate bond) is considered a fantastic financial instrument in terms of funding for the non-financial firms in the Brazilian market. The intermediation would be done in the capital market instead of through the commercial banks. The key issue for the development of this market is the financial engineering involving the credit risk (chance that the corporate issuer can default on its debt obligation). This paper proposes and tests a methodology to quantify this risk in a cross-section of Brazilian debentures. Our approach is based on Merton's (1974) asset pricing model that uses the Black-Schole's put option formula. The consequent optimization techniques allow us to infer the risk of debentures. By using a simple and low-cost model, we find a risk measure that is more conservative than the usual VaR (value at risk). Thus, we present a methodology for obtaining the optimum portfolio composed of debentures subject to the default risk.
\end{abstract}

\section{KEYWORDS}

credit risk, corporate bonds, maximum loss, Merton's Asset Pricing Model

\section{JEL CLASSIFICATION}

GI2, G32

\footnotetext{
* Santander Asset Management - Risco de Mercado - Endereço para contato: Av. das Nações Unidas, I290I, Torre Norte, 19o andar - cep: 04578-900 - São Paulo, Brasil. E-mail: acgodoi@santander.com.br.

* Universidade de São Paulo, Departamento de Economia. Endereço para contato: Av. Professor Luciano Gualberto, 908 - cep: 05508-010 São Paulo, Brasil. E-mail: pyoshino@usp.br. webpage: www.econ.fea.usp/joe.

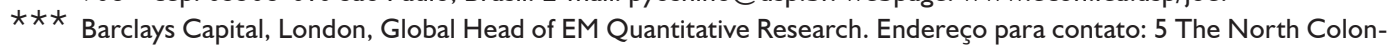
nade, Ist floor, Canary Wharf, London, El4 4BB, UK. E-mail: rogerio.oliveira@barcap.com.

(Recebido em março de 2007. Aceito para publicação em outubro de 2007).
} 


\section{INTRODUÇÃO}

O risco de crédito pode ser definido como o risco ao qual a instituição credora está exposta caso alguma de suas contrapartes venha a falhar no cumprimento de suas obrigações contratuais de crédito. Apesar da dimensão que esse tipo de risco representa para as instituições financeiras, apenas recentemente novas técnicas e idéias surgiram de maneira a contribuir de forma relevante para o desenvolvimento do tema.

O uso da modelagem neste campo, por exemplo, é um evento recente. Para o tratamento do risco de crédito de clientes corporativos, por exemplo, os modelos mais importantes começaram a surgir após a revisão do acordo de capital da Basiléia desenvolvido pelo BIS (Bank for International Settlements), o qual propõe métodos mais sofisticados para a alocação de capital contra exposições de crédito comercial, de varejo, interbancário e soberano.

Para contribuir na pesquisa na área de modelagem de crédito, este trabalho apresenta um método para a determinação do risco para tomadores corporativos, usando uma abordagem mais voltada para o mercado ou para o efeito das variações de preços causadas por eventos de crédito sobre o retorno da carteira. Aqui, assume-se que os preços e a dinâmica do mercado antecipam riscos, turbulências e dificuldades que possam ocorrer no futuro da firma emissora de um título. Vale observar que a abordagem de mercado é viável para o problema que iremos tratar, já que o risco será calculado para uma carteira composta por títulos corporativos, mais precisamente por debêntures.

O uso de debêntures como instrumentos de crédito foi outro motivador para o desenvolvimento deste artigo. Este produto vem se tornando cada vez mais atrativo, seja do ponto de vista do emissor - por causa da flexibilidade de sua estrutura e do custo mais baixo perante outras opções de captação de recursos, como os empréstimos -, seja do ponto de vista do investidor - por ser uma boa opção de diversificação de risco, com sua gama extensa de indexadores, e de planejamento de fluxo de caixa, por sua estrutura flexível de pagamentos de juros e amortizações de principal. Além disso, o mercado secundário de debêntures vem aumentando sua liquidez nos últimos meses, principalmente após a iniciativa da Associação Nacional das Instituições do Mercado Aberto (Andima) de divulgar diariamente uma pesquisa de taxas indicativas para várias emissões. O volume médio diário de janeiro a outubro de 2005 , por exemplo, apresentou um crescimento de 75\% com relação ao volume médio diário de 2004. 


\section{TABELA I - VOLUME MÉDIO DIÁRIO NO MERCADO SECUNDÁRIO DE DEBENTURES}

\begin{tabular}{lc}
\hline Ano & Volume médio diário ( $\mathrm{R} \$$ milhões) \\
\hline 2003 & 56,09 \\
2004 & 44,61 \\
2005 (janeiro a outubro) & 78,39 \\
\hline
\end{tabular}

Fonte: Sistema Nacional de Debêntures

Outro fator de motivação para a realização deste trabalho foi a utilização de um modelo de risco alternativo, que supera a limitação imposta pelo alto custo computacional gerado na implementação de simulações de Monte Carlo, necessária caso tratássemos o problema segundo uma abordagem tradicional de value at risk (VaR), uma vez que a distribuição de probabilidades do retorno do portfolio de crédito apresenta assimetria e caudas grossas. No nosso problema, os fatores de risco, - os valores de mercado dos ativos das emissoras das debêntures, foram obtidos a partir do Modelo de Merton (1974) para o apreçamento de títulos corporativos. Para o cálculo do risco do portfolio, adotou-se a abordagem denominada Perda Máxima (PM). Neste caso, não se assumiu nenhuma distribuição de probabilidades do retorno do portfolio e resolveu-se um problema de minimização da função retorno $(P \mathcal{E} L)$ da carteira.

Uma conseqüência interessante da utilização do método (PM) é a obtenção de uma medida de risco mais conservadora do que a obtida caso usássemos o conceito de (VaR). O leitor é remetido a Studer (1995) caso se interesse em verificar a comprovação formal deste resultado.

Obter uma medida quantitativa de risco agregado a partir da função (PM) é nosso objetivo principal, mas não o único. Como a determinação de (PM) é um problema de minimização de risco por média-variância, também iremos apresentar a otimização do portfolio a partir de uma composição que corresponda à fronteira eficiente.

Para uma melhor exposição das ferramentas usadas e dos resultados obtidos na resolução dos problemas citados, este artigo está organizado da seguinte maneira: a seção 2 apresenta a base teórica do trabalho, com uma descrição dos modelos de Merton (1974) e de Perda Máxima (PM). Na seção 3, são descritos os dados e as ferramentas metodológicas utilizadas. Já na seção 4 , é apresentada a estimação dos modelos. $\mathrm{Na}$ seção 5, são apresentadas as conclusões. Por fim, os anexos I e II apresentam o desenvolvimento dos modelos para a determinação do risco de crédito da carteira e de sua composição ótima. 


\section{OS MODELOS: PERDA MÁXIMA E MERTON ESTENDIDO}

Um dos objetivos deste trabalho é testar uma metodologia alternativa ao já amplamente conhecido método Value at Risk (VaR) para a mensuração do risco de uma carteira de debêntures. Em outras palavras, agregaremos o risco de crédito do portfolio de teste a partir da minimização da função Retorno, ou determinação da função Perda Máxima (PM), a qual descreveremos a seguir. Em seu artigo, Studer, (1995) utiliza este método para calcular o risco de portfolios compostos por ativos não-lineares e comprova seu maior conservadorismo com relação à metodologia VaR. ${ }^{1}$

Podemos definir a perda máxima como a rentabilidade mínima que a carteira de debêntures pode atingir no horizonte de tempo de um ano, dado um nível de confiança. Em termos do valor da carteira de debêntures, podemos defini-la como a pior perda financeira que a carteira pode sofrer em termos relativos nesse período. De maneira mais formal, a função "perda máxima" (PM) pode ser definida como a máxima perda:

i) Tal que os fatores de risco permaneçam confinados em uma região de confiança $(A t), \operatorname{Pr}(\mathfrak{w} \mid \mathfrak{w} \in A t)=\alpha$, onde $(\alpha)$ é o nível de confiança.

ii) Para um horizonte de tempo $(t)$.

Podemos resumir essa definição com a equação:

$$
\begin{aligned}
& P M=\min v(w) \\
& \text { s.t } w \in A_{t}, \quad \text { onde } \operatorname{Pr}\left(A_{t}\right)=\alpha
\end{aligned}
$$

onde $(v(w))$ é a função P\&L (profit and loss) e (w) é o fator de risco. Escolhendo uma região de confiança $\left(A_{t}\right)$ adequada, podemos reescrever o problema de minimização acima como:

$$
\begin{aligned}
& P M=\min v(w) \\
& \text { s.t } \quad(w-\mu)^{\mathrm{T}} \Sigma^{-1}(w-\mu) \leq \chi_{1 \%, D F}^{2}
\end{aligned}
$$

O leitor interessado pode conferir a obtenção da região de confiança $\left(A_{t}\right)$ em Studer (1995). Outro resultado demonstrado pelo mesmo autor do artigo é o fato de que a medida de risco (PM) será sempre mais conservadora do que o (VaR). Assim, com a troca do (VaR) por (PM) no nosso trabalho, conseguimos uma medida mais conser-

1 Nos casos em que a função retorno do portfolio é linear com relação aos fatores de risco, Studer (1995) demonstra que as duas metodologias coincidem. 
vadora de risco, além de evitarmos o custo computacional da realização de simulações de Monte Carlo.

Outro modelo que serve de base para o desenvolvimento deste artigo é o de Merton (1974) para risco de crédito, o qual inspirou uma vasta teoria e um amplo desenvolvimento do tema de apreçamento de títulos corporativos. Em seu trabalho, Merton propôs uma metodologia que se baseia na análise da capacidade de pagamento da empresa emissora do título privado. O modelo simplifica a estrutura do balanço da empresa emissora da debênture como se o seu passivo fosse composto apenas da dívida (debênture) a ser apreçada. Assim, na data de vencimento do passivo, a empresa terá capacidade de honrar seu compromisso se possuir ativos suficientes $(V)$ para pagar a dívida $(F)$. Para o detentor da debênture, é importante que $F$ seja maior que $V$ no vencimento para que ele receba o valor que emprestou no início da operação. Caso isso ocorra, seu payoff será de $F$ (valor de face da dívida). Caso não ocorra, seu payoff será equivalente à magnitude dos ativos restantes da empresa. Podemos expressar esse payoff no vencimento como:

$$
\operatorname{Payoff}_{T}=F-E\{\operatorname{Max}[0, F-V]\}
$$

Analisando a função payoff (3), Merton concluiu que ser o detentor da dívida é equivalente a vender uma opção de venda (put) no valor dos ativos do tomador, e o preço de exercício da opção equivale ao valor de face do empréstimo. Esse raciocínio considera que se o valor de mercado dos ativos da empresa $(V)$ no vencimento for menor do que o passivo da empresa $(F)$, representado pelo valor de face do empréstimo, a empresa devedora entra em default. Utilizando o modelo de Black-Scholes para o apreçamento de opções européias, chega-se ao resultado proposto por Merton, resumido pela equação a seguir:

$$
P(0, T)=V_{0} N\left(-d_{1}\right)+F e^{-r T} N\left(d_{2}\right)
$$

onde:

$$
\begin{aligned}
& d_{1}=\frac{\ln \left(\frac{V_{0}}{F}\right)+\left(r+\sigma_{v}{ }^{2} / 2\right) t}{\sigma_{v} \sqrt{t}} \\
& d_{2}=d_{1}-\sigma_{v} \sqrt{t}
\end{aligned}
$$

$(v)$ é a taxa livre de risco, $\left(\sigma_{v}\right)$ é a volatilidade do ativo, $N($.) é a função normal padrão acumulada e $T$ é o prazo da operação. 
Uma importante limitação deste modelo é que ele só pode ser aplicado em títulos do tipo "zero-cupom". Como a carteira a ser testada é composta por debêntures, precisamos de um modelo que contemple a presença dos pagamentos de juros periódicos que fazem parte da estrutura das debêntures. Para isso, utilizaremos uma extensão do modelo de Merton (1974), testado por Eom, Helwege e Huang (2003). Nesta versão do modelo, o título que possui pagamento de juros intermediários é considerado um portfolio de títulos zero-cupom, e cada um deles é apreçado pela versão clássica do Modelo de Merton (1974). O somatório dos fluxos resulta em uma fórmula que será descrita a seguir.

Consideremos uma debênture, sujeita a risco de crédito do emissor, com prazo de vencimento $(T)$, com valor de face unitária e que paga um cupom semi-anual a uma taxa anual igual a $(c)$. Sejam $\left(T_{i}\right) ; i=1, \ldots, T$; as datas de pagamento de cupom. No modelo de Eom, Helwege e Huang (2003) (Merton estendido), o preço de um título sujeito a default pode ser escrito como:

$$
\begin{aligned}
P(0, T)= & \sum_{i=1}^{T-1} D\left(0, T_{i}\right)\left[(c / 2) N\left(d_{2}\left(c / 2, T_{i}\right)\right)+V_{0} N\left(-d_{1}\left(c / 2, T_{i}\right)\right) D^{-1}\left(0, T_{i}\right)\right] \\
& +D(0, T)\left[(1+c / 2) N\left(d_{2}(1+c / 2, T)\right)+V_{0} N\left(-d_{1}(1+c / 2, T)\right) D^{-1}(0, T)\right]
\end{aligned}
$$

onde $\left(D\left(0, T_{i}\right)\right)$ denota o valor presente de um bônus "zero-cupom" com vencimento em $\left(T_{i}\right), N($.$) representa a função normal padrão acumulada e$

$$
\begin{aligned}
& d_{1}(x, t)=\frac{\ln \left(\frac{V_{0}}{x}\right)+\left(D(0, t)^{-1}+\sigma_{v}{ }^{2} / 2\right)}{\sigma_{v} \sqrt{t}} ; \\
& d_{2}(x, t)=d_{1}(x, t)-\sigma_{v} \sqrt{t}
\end{aligned}
$$

onde $\left(\sigma_{\mathrm{V}}\right)$ é a volatilidade dos ativos da empresa. Essa é a equação que usaremos para o apreçamento das debêntures que utilizaremos no nosso problema de otimização.

As aplicações teóricas dos modelos apresentados para a determinação do risco de crédito da carteira e da composição ótima estão desenvolvidas nos anexos I e II. Para o cálculo do risco, resolve-se a equação:

$$
\begin{aligned}
& P M=\min _{v} R T\left(V_{1}\right) \\
& \text { s.a. } \quad(v-\mu)^{T} \Sigma^{-1}(v-\mu) \leq \chi_{1 \%, N}^{2}
\end{aligned}
$$


onde:

$$
\begin{aligned}
& R T=\text { Retorno Total }=\sum_{i} \alpha_{i}\left[\frac{P_{i}(1, T)}{P_{i}(0, T)}-1\right] \\
& v=\ln \left(\frac{V_{1}}{V_{0}}\right)
\end{aligned}
$$

e onde $P_{i}(t, T)$ são os preços de cada debênture (i) obtidos da equação (6) para $t=0 \mathrm{e}$ $t=1 ;\left(\alpha_{\mathrm{i}}\right)$ é o peso de cada debênture (i) na carteira; $(\Sigma)$ é a matriz de covariância; e $\left(\chi_{1 \%, N}^{2}\right)$ é o quantil $1 \%$ da distribuição qui-quadrada com $(N)$ graus de liberdade.

Já a composição ótima da carteira é obtida a partir da resolução simultânea de dois problemas de otimização. O primeiro para o cálculo do risco:

$$
\begin{aligned}
& P M=\min _{v} R T\left(V_{1}\right) \\
& \text { s.a. } \quad(v-\mu)^{T} \Sigma^{-1}(v-\mu) \leq \chi_{1 \%, N}^{2}
\end{aligned}
$$

onde $(v)$ é o vetor de log-retornos dos ativos, $(\Sigma)$ é a matriz de co-variância, $\left(\chi_{1 \%, N}^{2}\right)$ é o quantil $1 \%$ da distribuição qui-quadrada com $(N)$ graus de liberdade e $R T$ é o retorno total da carteira, expresso pela equação (8).

E o segundo para a minimização deste risco para um valor fixo de $(\mu)$ :

$$
\begin{gathered}
\min _{\alpha} P M \\
\text { s.t. } \quad \sum_{i} \alpha_{i} r_{i}=\mu \\
\sum_{i} \alpha_{i}=1
\end{gathered}
$$

onde $\left(r_{i}\right)$ são os retornos esperados de cada debênture para o horizonte de tempo de um ano e $(\mu)$ é o retorno esperado do portfolio para o mesmo prazo.

\section{DESCRIÇÃO DOS DADOS E METODOLOGIA}

Foram avaliadas 32 emissões que faziam parte em 16/10/2004 do conjunto de debêntures com taxas referenciais divulgadas diariamente pela Andima. Dentro deste universo de títulos, foram selecionados os papéis que fariam parte da carteira de análise. Os critérios desta seleção serão descritos a seguir: 
i) Prazo: Dado o horizonte de tempo de 1 ano escolhido para a nossa análise, foram descartadas as debêntures com maturidade menor do que este prazo.

ii) Estrutura: Foram selecionadas emissões que possuem fluxos de pagamento de juros e principal semelhantes. Foram descartadas aquelas corrigidas por correção monetária.

iii) Taxas: Foram descartadas aquelas emissões cujos prêmios de risco ou spreads derivados das taxas referenciais possuem volatilidade histórica excessiva ou incoerente com o perfil de risco da empresa emissora. Alguns exemplos são as debêntures cuja referência "livre de risco" possui pouca liquidez e, com isso, um certo grau de subjetividade na obtenção de seu preço. Há também o problema da diversidade de ratings atribuídos por agências distintas à mesma emissão. Segundo Saito e Sheng (2005), essa discrepância afeta o valor do spread e pode causar uma volatilidade espúria. Em função deste critério, quase todas as emissões indexadas ao IGPM foram descartadas.

Das 32 debêntures presentes na pesquisa, 17 eram atreladas ao CDI e 15 indexadas ao IGPM. Pelo critério (iii), apenas a emissão CMIG21 foi considerada deste segundo grupo. Considerando que os testes foram realizados em junho de 2005, o critério (i) de prazo mínimo de 1 ano (incluindo repactuações) excluiu oito emissões do grupo de 17 indexadas ao CDI: BRTO13, TMAR 14, TMAR24, VTRF13, VTRF23, CPLE22, MRLMll e TNLP1l. Ainda neste grupo, a emissão BRKMll foi recomprada durante o período de coleta de preços e a emissão CSNAl3 foi descartada por possuir o mesmo prazo e mesmo emissor que a emissão CSNA12, considerada na carteira de testes. Assim, no final, foram escolhidas oito emissóes, cujas características podem ser vistas na Tabela 2. Para estas oito emissóes, foram coletadas as taxas referenciais da Andima $^{2}$ para o período de 18/10/2004 a 16/06/2005. A partir das taxas, foram determinados os preços unitários (PU) de mercado para cada uma das emissóes, segundo as regras especificadas no manual de apreçamento CONFERE de debêntures (ver ASSOCIAÇÃO NACIONAL DAS INSTITUIÇÕES DO MERCADO ABERTO, 2005) desenvolvido pela Associação.

Com os preços e características dos papéis em mãos e utilizando a fórmula de Merton estendida (6), foi possível a obtenção da série histórica dos valores de mercado dos ativos das empresas emissoras das debêntures. Para isso, utilizou-se o mesmo procedimento do modelo KMV (CROSBIE; BOHN, J., 2005), que faz uso de um método recursivo para a obtenção da série e da volatilidade dos retornos dos ativos. ${ }^{3} \mathrm{~A}$ volati-

2 A pesquisa de taxas da Andima é divulgada desde maio de 2004, porém esta só se tornou mais madura com a adesão de mais contribuidores, o que ocorreu alguns meses depois.

3 A fórmula utilizada para o cálculo dos retornos é dada pelo logaritmo neperiano da razão entre os valores atual e imediatamente anterior, de forma a se obter consistência com a hipótese do Modelo de 
lidade, definida como o desvio padrão de cada série histórica dos retornos dos valores de mercado dos ativos das empresas emissoras das debêntures, foi determinada de maneira implícita. Para cada emissão, adotou-se uma estimativa inicial dessa volatilidade e, por meio da equação de Merton estendida, determinou-se uma primeira versão da série histórica de retornos dos valores de mercado do ativo da empresa emissora. A volatilidade da série resultante de retornos foi usada como entrada para a próxima iteração do procedimento, que, por sua vez, calculou uma nova série de valores de retornos dos ativos. O método seguiu até que se encontrasse uma convergência entre as estimativas e os valores reais das volatilidades das séries. ${ }^{4}$

\section{TABELA 2 - CARACTERÍSTICAS DAS DEBÊNTURES SELECIONADAS NA PESQUISA DIÁRIA DE PREÇOS DA ANDIMA}

\begin{tabular}{llcccc}
\hline Código & Nome do Emissor & $\begin{array}{c}\text { Data de } \\
\text { Emissão }\end{array}$ & Vencimento & Indexador & Cupom \\
\hline CSNA12 & CSN & $01 / 12 / 2003$ & $01 / 12 / 06$ & CDI & $107 \%$ CDI \\
ALLG13 & $\begin{array}{l}\text { AMÉRICA LATINA } \\
\text { LOGÍSTICA }\end{array}$ & $01 / 06 / 2004$ & $01 / 06 / 07$ & CDI & $110 \%$ CDI \\
LAME12 & LOJAS AMERICANAS & $01 / 01 / 2004$ & $01 / 01 / 07$ & CDI & CDI + 0.90\% \\
NVML12 & NOVAMARLIM & $01 / 12 / 2001$ & $01 / 12 / 06$ & CDI & CDI + 1.10\% \\
BRT014 & BRASIL TELECOM & $05 / 07 / 2004$ & $05 / 07 / 09$ & CDI & CDI + 1.15\% \\
PALF12 & CPFL PAULISTA & $01 / 07 / 2004$ & $01 / 07 / 09$ & CDI & $109 \%$ CDI \\
SBES16 & SABESP & $01 / 09 / 2004$ & $01 / 09 / 07$ & CDI & CDI + 1.75\% \\
CMIG21 & CEMIG & $01 / 11 / 2001$ & $01 / 11 / 06$ & IGPM & IGPM + 12.70\%
\end{tabular}

Para que se possa utilizar corretamente o modelo de Merton (1974), as séries dos logretornos devem possuir distribuição de probabilidades Normal. Além disso, uma das hipóteses do modelo Perda Máxima (PM) de cálculo de risco é que os fatores de risco devem possuir distribuição conjunta Normal multivariada. Com base nisso, usamos testes estatísticos, como o Jarke-Bera e gráficos quantil-quantil, em cada uma das séries para verificarmos para quais delas podemos aceitar a hipótese nula de distribuição Normal. Verificamos, também, os valores de assimetria e curtose das distribuições.

Merton (1974) de que a série de log-retornos do ativo subjacente possui distribuição Normal.

4 O modelo KMV (CROSBIE; BOHN, 2005) adota a hipótese de volatilidade constante para as séries, o que não necessariamente reflete a realidade e constitui-se em um ponto fraco do método. 
Com isso, pudemos concluir que para apenas seis das oito séries escolhidas temos um p-valor razoável no teste Jarke-Bera para a adoção da hipótese de normalidade. As seis emissões selecionadas foram: CSNA12, ALLG13, LAME12, NVML12, BRTOl4 e SBES16. ${ }^{5}$

A partir das séries históricas de (PUs), foi possível chegar implicitamente aos valores de média, volatilidade e à matriz de co-variância das séries dos log-retornos dos ativos, conforme foi descrito anteriormente. Para a determinação do risco de crédito do portfolio, resolveu-se o sistema de equações descrito no anexo I para uma composição uniforme de debêntures. As informações sobre esta composição e sobre média e variância das séries dos retornos dos ativos podem ser conferidas nas Tabelas 3, 4 e 5 .

\section{TABELA 3 - COMPOSIÇÃO DA CARTEIRA TESTE}

\begin{tabular}{lcccc}
\hline Emissões & Valor investido $(\mathrm{R} \$)$ & Composição $(\%)$ & $\mathrm{P}(0, \mathrm{~T})$ & \multicolumn{1}{c}{$\mathrm{V}^{*}$} \\
\hline CSNA12 & 1.000 .000 & $16,67 \%$ & $10.118,96$ & $7.877,60$ \\
ALLG13 & 1.000 .000 & $16,67 \%$ & $10.181,10$ & $7.360,87$ \\
LAME12 & 1.000 .000 & $16,67 \%$ & $10.858,02$ & $7.773,67$ \\
NVML12 & 1.000 .000 & $16,67 \%$ & $2.220,11$ & $1.567,00$ \\
BRT014 & 1.000 .000 & $16,67 \%$ & $10.898,02$ & $5.693,77$ \\
\hline SBES16 & 1.000 .000 & $16,67 \%$ & $1.062,85$ & 691,84 \\
\hline
\end{tabular}

* Preço da debênture com vencimento em $T$ no instante inicial $t=0$; ** Valor de mercado dos ativos da empresa emissora no instante inicial $t=0$.

TABELA 4-MÉDIAS E VOLATILIDADES ANUALIZADAS DOS LOG-RETORNOS DOS ATIVOS (PERÍODO DE 18/10/2004 A 16/06/2005)

\begin{tabular}{lcc}
\hline Emissões & Volatilidades dos ativos & Médias dos ativos \\
\hline CSNA12 & $3,69 \%$ & $20,18 \%$ \\
ALLG13 & $5,69 \%$ & $23,21 \%$ \\
LAME12 & $3,54 \%$ & $20,91 \%$ \\
NVML12 & $3,11 \%$ & $18,49 \%$ \\
BRT014 & $11,04 \%$ & $28,17 \%$ \\
SBES16 & $6,32 \%$ & $26,37 \%$ \\
\hline
\end{tabular}

5 A hipótese de normalidade é exigida para que haja coerência com as hipóteses do Modelo de Merton (1974). Todavia, as duas emissões descartadas poderiam ser aproveitadas e suas distribuições de probabilidades estimadas por algum método alternativo, como o Generalized Method of Moments (GMM). 


\section{TABELA 5 - MATRIZ DE CO-VARIÂNCIA (PERÍODO DE 18/10/2004 A $16 / 06 / 2005)$}

\begin{tabular}{lcccccc}
\hline & CSNA12 & ALLG13 & LAME12 & NVML12 & BRTO14 & SBES16 \\
\hline CSNA12 & $0,13 \%$ & $0,19 \%$ & $0,13 \%$ & $0,10 \%$ & $0,34 \%$ & $0,20 \%$ \\
ALLG13 & $0,19 \%$ & $0,32 \%$ & $0,18 \%$ & $0,14 \%$ & $0,51 \%$ & $0,30 \%$ \\
LAME12 & $0,13 \%$ & $0,18 \%$ & $0,12 \%$ & $0,10 \%$ & $0,32 \%$ & $0,19 \%$ \\
NVML12 & $0,10 \%$ & $0,14 \%$ & $0,10 \%$ & $0,10 \%$ & $0,25 \%$ & $0,15 \%$ \\
BRT014 & $0,34 \%$ & $0,51 \%$ & $0,32 \%$ & $0,25 \%$ & $1,21 \%$ & $0,57 \%$ \\
SBES16 & $0,20 \%$ & $0,30 \%$ & $0,19 \%$ & $0,15 \%$ & $0,57 \%$ & $0,40 \%$ \\
\hline
\end{tabular}

\section{ESTIMAÇÃO DO MODELO}

Sabendo que o retorno agregado do portfolio pode ser expresso por:

$$
\text { Retorno do portfolio }=\sum_{i} w_{i} r_{i}
$$

onde $\left(\mathfrak{w}_{i}\right)$ são os pesos de cada debênture $(i)$ na composição da carteira e $\left(r_{i}\right)$ são os retornos esperados para cada debênture (i) no horizonte de tempo de um ano, procurou-se determinar o valor mínimo deste retorno variando os valores dos ativos $\left(V_{1, i}\right)$ de forma que o retorno agregado destes ativos, expresso por:

$$
\text { Retorno dos ativos }=\sum_{i} w_{i} v_{i}
$$

onde $\left(v_{i}\right)$ são os log-retornos dos ativos no horizonte de tempo de um ano, fosse igual ao quantil $1 \%$ da distribuição qui-quadrada para 6 graus de liberdade, cujo valor é igual a 16,81. Com base nisso, obtivemos os resultados expressos na Tabela 6:

\section{TABELA 6 - RESULTADOS DO PRIMEIRO PROBLEMA DE OTIMIZAÇÃO}

\begin{tabular}{lcrcc}
\hline Emissões & Composição & $\mathrm{V}(\mathrm{t}=1)^{*}$ & Ret $\mathrm{V}^{* *}$ & Ret PU*** \\
\hline CSNA12 & $16,67 \%$ & 8327,141 & $-14,63 \%$ & $8,6 \%$ \\
ALLG13 & $16,67 \%$ & 7476,627 & $-21,65 \%$ & $7,8 \%$ \\
LAME12 & $16,67 \%$ & 8338,526 & $-13,89 \%$ & $10,1 \%$ \\
NVML12 & $16,67 \%$ & 1685,5 & $-11,20 \%$ & $10,0 \%$ \\
BRT014 & $16,67 \%$ & 4968,131 & $-41,80 \%$ & $6,9 \%$ \\
SBES16 & $16,67 \%$ & 708,8347 & $-23,95 \%$ & $8,5 \%$ \\
\hline
\end{tabular}

* Valor dos ativos no horizonte de tempo $t=1$ ano; ${ }^{*} \log$-retorno dos ativos; *** retorno mínimo de cada debênture. 6

Obs: Retorno acumulado dos ativos (equação 12) $=16,81$. Quantil 1\% da distribuição qui-quadrada para 6 graus de liberdade $=16,81$. Retorno agregado mínimo (equação 11 ) =8,6\%.

$6 \operatorname{ret} P U=P U(t=1) / P U(t=0)-1$ 
O programa de otimização fornece um valor positivo para a rentabilidade mínima da carteira, o que contradiz a idéia de "perda". Mas este valor, apesar de positivo, fica muito aquém da expectativa de rentabilidade para a carteira no prazo. Portanto, nos pareceu mais coerente representar a perda máxima como a diferença entre a variação obtida do programa de otimização e a variação esperada da taxa de juros, também chamada de "custo de carregamento". Assim, descontando do retorno mínimo um valor esperado para o custo de carregamento de $18,29 \%,{ }^{7}$ estimado pelo valor da taxa de juros em reais a termo para o prazo de um ano, a perda máxima do portfolio é dada por:

$$
\text { PERDA MÁXIMA }=8,65 \%-18,29 \%=-9,64 \%
$$

Verificaremos agora como se comporta o risco quando alteramos a composição da carteira, mantendo inalterado o valor esperado do retorno do portfolio. Os resultados referentes a esse exercício podem ser vistos na Tabela 7:

\section{TABELA 7 - RETORNOS MÍNIMOS PARA DIVERSAS COMPOSIÇÕES COM $\mu$ CONSTANTE}

\begin{tabular}{lcccc}
\hline & Composição 1 & Composição 2 & Composição 3 & Composição 4 \\
\hline CSNA12 & $10,46 \%$ & $16,67 \%$ & $9,96 \%$ & $13,59 \%$ \\
ALLG13 & $27,99 \%$ & $16,67 \%$ & $10,59 \%$ & $20,45 \%$ \\
LAME12 & $10,46 \%$ & $16,67 \%$ & $18,64 \%$ & $14,91 \%$ \\
NVML12 & $24,36 \%$ & $16,67 \%$ & $18,78 \%$ & $19,66 \%$ \\
BRT014 & $9,14 \%$ & $16,67 \%$ & $20,90 \%$ & $11,38 \%$ \\
SBES16 & $17,59 \%$ & $16,67 \%$ & $21,13 \%$ & $20,01 \%$ \\
Ret. Esperado & $19,48 \%$ & $19,48 \%$ & $19,48 \%$ & $19,48 \%$ \\
Retorno Mínimo & $\mathbf{8 , 6 2} \%$ & $\mathbf{8 , 6 5 \%}$ & $\mathbf{8 , 6 7 \%}$ & $\mathbf{8 , 7 0 \%}$ \\
PERDA MÁXIMA & $-\mathbf{9 , 6 7 \%}$ & $-\mathbf{- 9 , 6 4 \%}$ & $-\mathbf{9 , 6 2} \%$ & $\mathbf{- 9 , 5 9 \%}$ \\
\hline
\end{tabular}

Verificando este comportamento do risco, é natural que se queira determinar qual a composição ótima da carteira, que nos leva ao menor risco dado um mesmo valor de retorno. A alocação ótima pode ser obtida com a resolução simultânea dos dois problemas de minimização expressos pelas equações (P1) e (P2) da seção 2 e cuja solução é apresentada no anexo II. Os resultados podem ser conferidos na Tabela 8.

7 Valor obtido a partir das cotações de fechamento do dia para contratos de Futuro DIl. Fonte: BM\&F. 
TABELA 8 - COMPOSIÇÃO ÓTIMA DA CARTEIRA

\begin{tabular}{lcc}
\hline emissões & composição & retornos* \\
\hline CSNA12 & $0,01 \%$ & $8,78 \%$ \\
ALLG13 & $10,34 \%$ & $8,17 \%$ \\
LAME12 & $24,52 \%$ & $10,19 \%$ \\
NVML12 & $18,66 \%$ & $10,04 \%$ \\
BRT014 & $8,26 \%$ & $7,85 \%$ \\
SBES16 & $38,21 \%$ & $7,61 \%$ \\
\hline
\end{tabular}

* retorno individual mínimo de cada debênture.

Obs: Retorno esperado do portfolio $=19,48 \%$.

Descontando o custo de carregamento estimado de $18,29 \%$ do valor do retorno, a perda máxima do portfolio é dada por:

$$
\text { PERDA MÁXIMA }=8,78 \%-18,29 \%=-9,51 \%
$$

Generalizando o problema e implementando o programa para outras composições que resultem em valores de $(\mu)$ distintos, chegamos aos resultados expostos na Tabela 9:

TABELA 9 - COMPOSIÇÓES ÓTIMAS PARA CARTEIRAS COM $\mu$ DISTINTOS

\begin{tabular}{lccccc}
\hline & Comp. Ótima 1 & Comp. Ótima 2 & Comp. Ótima 3 & Comp. Ótima 4 & Comp. Ótima 5 \\
\hline CSNA12 & $0,01 \%$ & $4,46 \%$ & $11,68 \%$ & $16,24 \%$ & $19,96 \%$ \\
ALLG13 & $10,34 \%$ & $10,85 \%$ & $11,74 \%$ & $12,37 \%$ & $12,88 \%$ \\
LAME12 & $24,52 \%$ & $19,99 \%$ & $12,58 \%$ & $7,82 \%$ & $3,94 \%$ \\
NVML12 & $18,66 \%$ & $17,33 \%$ & $15,06 \%$ & $13,52 \%$ & $12,26 \%$ \\
BRT014 & $8,26 \%$ & $8,35 \%$ & $8,51 \%$ & $8,64 \%$ & $8,74 \%$ \\
SBES16 & $38,21 \%$ & $39,02 \%$ & $40,43 \%$ & $41,41 \%$ & $42,21 \%$ \\
$\mu$ & $19,48 \%$ & $19,58 \%$ & $19,68 \%$ & $19,78 \%$ & $19,88 \%$ \\
Ret. Mínimo & $\mathbf{8 , 7 8} \%$ & $\mathbf{8 , 6 7 \%}$ & $\mathbf{8 , 5 1 \%}$ & $\mathbf{8 , 4 0 \%}$ & $\mathbf{8 , 3 1 \%}$ \\
Perda Máx. & $\mathbf{- 9 , 5 1 \%}$ & $\mathbf{- 9 , 6 2 \%}$ & $-\mathbf{9 , 7 8 \%} \%$ & $-\mathbf{9 , 8 9 \%} \%$ & $-\mathbf{9 , 9 8 \%}$ \\
\hline
\end{tabular}

Usando os valores apresentados nas tabelas anteriores, obtemos a fronteira eficiente, expressa no Gráfico 1. 


\section{GRÁFICO I - FRONTEIRA EFICIENTE}

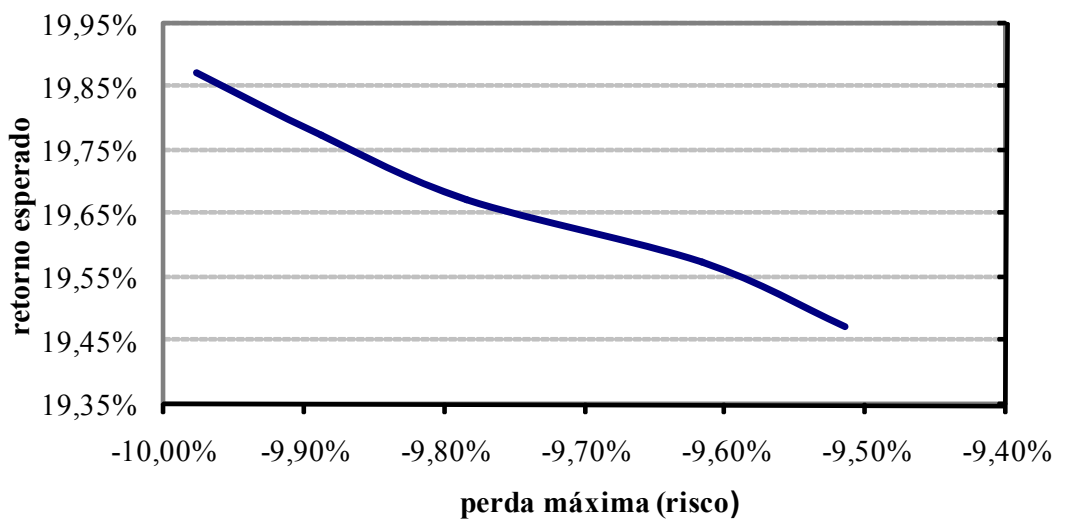

Do gráfico acima, podemos verificar a curva que identifica outras composições de mínima variância, para valores de retorno esperado do portfolio diferentes. Ela é condizente com a premissa de que para o alcance de retornos maiores, o investidor deve assumir riscos maiores.

Até o momento, conseguimos comprovar a eficácia e objetividade do método para a alocação da carteira ótima, cuja solução obtivemos com um sistema de apenas duas equações e duas incógnitas. Falta, também, comprovar empiricamente a outra vantagem deste método, que é obter uma medida de risco mais conservadora do que o value at risk (VaR) através da função perda máxima (PM). Além disso, apesar da obtenção de resultados coerentes, precisamos verificar se estes resultados condizem com a realidade.

Para a prova empírica do resultado apresentado em Studer (1995), de que a (PM) é mais conservadora do que o (VaR), repetimos o uso do modelo de Eom, Helwege e Huang (2003) (Merton estendido) para a verificação do comportamento histórico dos ativos das empresas, mas usamos os parâmetros obtidos nesta análise para simular cenários futuros para estes ativos por Monte Carlo. ${ }^{8}$ Com os cenários futuros estimados, foi possível o cálculo dos preços das debêntures no horizonte de tempo de um ano. Considerando a composição ótima obtida na resolução do problema anterior, chegamos a uma distribuição de probabilidades para o retorno agregado do portfolio no horizonte de tempo estipulado. Esta distribuição pode ser conferida no gráfico a seguir.

8 Fizemos 10.000 simulaçóes de cenários futuros para os ativos de cada empresa emissora, para cem passos no futuro, e consideramos a correlação histórica entre os ativos de forma a vincular as variáveis aleatórias geradas para as seis empresas emissoras. 


\section{GRÁFICO 2 - HISTOGR AMA PARA A FUNÇÃO RETORNO DO PORTFOLIO}

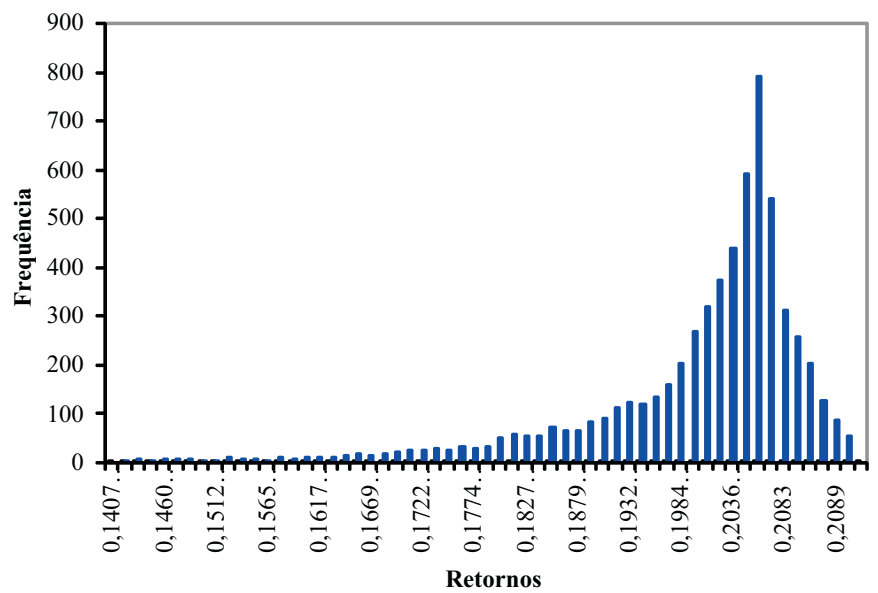

\begin{tabular}{|lr} 
Mean & $20,27 \%$ \\
Standard Deviation & $1,05 \%$ \\
Kurtosis & 12,58 \\
Skewness & $-2,94$ \\
\hline
\end{tabular}

O valor de retorno correspondente ao quantil 1\% da distribuição no Gráfico 2 é igual a $14,38 \%$. Descontando o custo de carregamento estimado de 18,29\% do valor do retorno, a perda máxima do portfolio calculada pelo (VaR) é dada por:

\section{PERDA MÁXIMA $=14,38 \%-18,29 \%=-3,94 \%$}

Comparando com o valor obtido pelo método (PM), igual a $-9,51 \%$, comprovamos que (PM) é uma medida de risco mais conservadora.

Voltando agora as atenções para a validação do método proposto neste trabalho, procuramos tratar o problema segundo uma abordagem alternativa, ou seja, determinando o risco de crédito a partir de um modelo de default e não mais através de um modelo de mercado ou de spread. O modelo alternativo que adotaremos (ver SAUNDERS, 2002, p. 98), muito similar ao CreditRisk+ (CREDIT SUISSE FIRST BOSTON INTERNATIONAL, 1997), trabalha com dois estados possíveis: default / não default. Nestes estados, a variável "perda de crédito" pode assumir os valores zero no estado "não default" e $p$ no estado "default". A variável aleatória "perda de crédito" é função de três variáveis: exposição, severidade e probabilidade de default; seu valor esperado e sua variância são expressos pelas equações a seguir:

$$
\begin{aligned}
& E[\text { perda }]=p_{d} \times E[\exp \text { osição } \times \text { severidade }]+\left(1-p_{d}\right) \times 0 \\
& =p_{d} \times E[\exp \text { osição }] \times E[\text { severidade }]=p_{d} \times e \times s \\
& \operatorname{var}(\text { perda })=e^{2} s^{2} p_{d}\left(1-p_{d}\right)+\sigma_{e}^{2} s^{2} p+\sigma_{s}^{2} e^{2} p+\sigma_{e}^{2} \sigma_{s}^{2} p
\end{aligned}
$$


Para aplicarmos essas definições nos nossos exemplos, devemos estimar os valores de cada uma das variáveis que definem a perda. Assim, utilizamos uma matriz de migração acumulada de ratings divulgada pela agência de classificação Moody's ${ }^{9}$ para estimar a probabilidade de default e utilizamos valores de taxas de recuperação também divulgadas pela agência Moody's (ver HAMILTON, 2004) para estimar a severidade. Os resultados podem ser conferidos nas Tabelas 10 e 11 a seguir. ${ }^{10}$

TABELA 10 - CARACTERÍSTICAS DAS EMISSÕES

\begin{tabular}{lrccl}
\hline & Exposição* $^{*}$ & Rating $^{* *}$ & Prazo aproximado & Espécie \\
\hline CSNA12 & 632 & A3 & 1 ano & Quirografária \\
ALLG13 & 620115 & A3 & 2 anos & Quirografária \\
LAME12 & 1470948 & A2 & 2 anos & Subordinada \\
NVML12 & 1119684 & Aa3 & 1 ano & Garantia Real \\
BRT014 & 495744 & Aa1 & 4 anos & Quirografária \\
SBES16 & 2292876 & A2 & 2 anos & Quirografária \\
\hline
\end{tabular}

TABELA 11 - PERDAS INDIVIDUAIS POR CRÉDITO ${ }^{11}$

\begin{tabular}{lccccrr}
\hline & $\begin{array}{c}\text { Taxa de } \\
\text { recuperação }\end{array}$ & $\begin{array}{c}\text { Taxa de } \\
\text { severidade }\end{array}$ & $\begin{array}{c}\text { Volatilidade da } \\
\text { severidade }\end{array}$ & $\begin{array}{c}\text { Probab. } \\
\text { default }\end{array}$ & $\begin{array}{c}\text { Perda } \\
\text { esperada }\end{array}$ & $\begin{array}{r}\text { Perda } \\
\text { Inesperada }\end{array}$ \\
\hline CSNA12 & $12,3 \%$ & $87,7 \%$ & $8,1 \%$ & $0,03 \%$ & 0,2 & 9,6 \\
ALLG13 & $12,3 \%$ & $87,7 \%$ & $8,1 \%$ & $0,21 \%$ & 1142,1 & 25001,9 \\
LAME12 & $12,3 \%$ & $87,7 \%$ & $8,1 \%$ & $0,08 \%$ & 1032,0 & 36628,1 \\
NVML12 & $12,3 \%$ & $87,7 \%$ & $8,1 \%$ & $0,00 \%$ & 98,2 & 9860,9 \\
BRT014 & $12,3 \%$ & $87,7 \%$ & $8,1 \%$ & $0,15 \%$ & 652,2 & 16897,6 \\
SBES16 & $12,3 \%$ & $87,7 \%$ & $8,1 \%$ & $0,08 \%$ & 1608,7 & 57094,9 \\
\hline
\end{tabular}

Até aqui, conseguimos determinar os valores de perda inesperada para cada debênture, mas precisamos de uma medida agregada ao portfolio. Para isso, precisaríamos considerar as correlações de default entre as contrapartes emissoras das debêntures, o que esbarra em um sério problema, ou seja, a falta de um banco de dados confiável para a apuração destas estatísticas. Como a nossa intenção é validar o modelo proposto pelo trabalho e não chegar a um valor exato de risco, agregamos o risco do portfolio segundo três cenários de correlação média entre as contrapartes. Para cada um destes

9 Dados de inadimplência coletados entre os anos de 1983 e 2004.

10 Segundo Saito e Sheng (2005), no caso de insolvência de uma empresa emissora é pouco provável que os credores recebam suas garantias devido à hierarquia prevista na Lei de Falências. Com base nisso, usaremos a taxa de recuperação mínima apresentada pela Moody's como referência para a severidade no modelo de default.

11 As estatísticas contidas na Tabela 11 foram extraídas da agência Moody's e refletem o mercado norteamericano de bônus corporativo.

12 Taxa de severidade $=1$ - taxa de recuperação. 
cenários, determinamos qual a volatilidade agregada da carteira. Com os valores de média e variância para cada cenário, parametrizamos a distribuição de probabilidades da função perda de crédito por uma distribuição "gama", assim como no método CreditRisk+. Com todas as variáveis necessárias em mãos, foi possível determinar o risco de crédito da carteira de debêntures para cada um dos cenários. Os resultados podem ser verificados na Tabela 12.

TABELA 12 - COMPARAÇÃO ENTRE OS RESULTADOS DA OTIMIZAÇÃO E DO MODELO DE DEFAULT

\begin{tabular}{cccc}
\hline & Retorno esperado* & Risco MD+ & Risco PM++ \\
\hline cenário 1 & $19,48 \%$ & $-4,63 \%$ & $-9,51 \%$ \\
cenário 2 & $19,48 \%$ & $-7,57 \%$ & $-9,51 \%$ \\
cenário 3 & $19,48 \%$ & $-9,04 \%$ & $-9,51 \%$ \\
\hline
\end{tabular}

* retorno esperado da carteira de debêntures para o horizonte $t=1$ ano; + risco medido pelo modelo de default; ++ risco medido pela otimização da função PM.

Pelos resultados exibidos na tabela anterior, vemos que a ordem de grandeza dos riscos medidos pelos dois modelos (PM) e (MD) é bem semelhante. Os valores não são muito próximos, uma vez que no modelo de default (MD) utilizamos valores de probabilidade de default divulgados pela agência Moody's, os quais não refletem a realidade brasileira e certamente não possuem relação com o comportamento histórico dos ativos obtidos a partir das séries de preços de debêntures adotadas neste trabalho.

Além disso, as taxas de severidade utilizadas também não refletem a realidade brasileira. Por vários problemas locais, inclusive de legislação, a parcela de crédito recuperada em um evento de default no Brasil é muito pequena. Portanto, é bastante razoável imaginar que as medidas de risco obtidas com o modelo de default sejam menos conservadoras do que as obtidas pela variável PM.

\section{CONCLUSÃO}

Foram apresentados neste trabalho dois problemas de otimização - um para o cálculo do risco de crédito para um portfolio de debêntures e outro para a obtenção da composição ótima da carteira, que minimiza o risco de crédito calculado anteriormente - e suas soluções. A principal motivação para se trabalhar com estes modelos foi a busca de uma alternativa simples para o cálculo do risco de crédito agregado. Para isso, procurou-se adaptar o modelo de Merton (1974) para o apreçamento de títulos corporativos e utilizou-se o método da otimização da perda máxima para a obtenção do risco. 
Com base nos resultados obtidos, pudemos concluir que a abordagem proposta é viável, objetiva, dado que não exige análises subjetivas das empresas envolvidas, e apresenta pouco custo computacional. O problema da alocação ótima do portfolio, por exemplo, impõe uma dupla otimização que, a princípio, parece complicar o problema. Porém, a solução se restringe a um simples sistema $2 \times 2$, cujas variáveis são os multiplicadores de Lagrange.

Além disso, vimos que o método apresenta outra vantagem com relação ao (VaR), por ser mais conservadora. Concluímos, também, que o método é factível, uma vez que fornece valores de risco semelhantes aos fornecidos por uma metodologia alternativa.

Algumas extensões e desenvolvimentos deste trabalho podem ser realizados, principalmente com a aplicação do método para quaisquer produtos de crédito e não somente para uma carteira de debêntures. Neste caso, poder-se-ia utilizar a idéia de "distance to default" utilizada no KMV (CROSBIE; BOHN, 2005) para a determinação de probabilidades de default, que seriam estimadas a partir dos dados de mercado de debêntures. Todavia, conforme comenta Securato (2003), para uma implementação correta do modelo, seria necessária uma extensa base de dados com informações de empresas e de situações de default, base esta que é de difícil estruturação no mercado brasileiro.

Também seria de grande utilidade a realização de algum teste de aderência ou backtest do modelo para uma amostra de dados não contemplados em sua calibração, de forma a avaliar a qualidade do modelo na previsão do risco de crédito.

\section{REFERENCIAS}

ASSOCIAÇÃO NACIONAL DAS INSTITUIÇÕES DO MERCADO ABERTO Andima. Confere de Debêntures: Metodologia. Rio de Janeiro, 2005. Disponível em: <http://www.andima.com.br>.

. Estudos Especiais; SND - Sistema Nacional de Debêntures. Rio de Janeiro, 1998. Disponível em: < http://www.debentures.com.br>.

BASLE COMMITTEE ON BANKING SUPERVISION. Credit Risk Modeling: Current Practices and Applications. Basle, 1999.

COSTA, O. L. V.; ASSUNÇÃO, H. G. V. Análise de risco e retorno em investimentos financeiros. Editora Manole, 2004.

CREDIT SUISSE FIRST BOSTON INTERNATIONAL. Credit Risk ${ }^{+}$- A Credit Risk Management Framework, 1997.

CROSBIE, P; BOHN, J. Modeling default risk. Moody's KMV Company, 2005. 
EOM, Y. H.; HELWEGE, J.; HUANG, J. Structural models of corporate bond pricing: an empirical analysis. Review of Financial Studies, 2003.

HAMILTON, D. et al. Default \& recovery rates of corporate bond issuers, Moodys's investors service - Global Credit Research, 2004. Disponível em: < http://www. moodys.com $>$.

HULL, J. Options, futures and other derivatives. $4^{\text {th }}$ Edition. Prentice Hall, 1993.

JAMES, B. R. Probabilidade: um curso em nivel intermediário. Associação Instituto Nacional de Matemática Pura e Aplicada, 2a Edição, 2002.

JOHNSON, R.; WICHERN, D. Applied multivarate statistical analysis. $5^{\text {th }}$ Edition. Prentice Hall, 2002.

JORION, P. Value at risk: the new benchmark for controlling market risk. Mc Graw Hill, 1997.

KEALHOFER, S. Quantifying credit risk II: debt valuation. AIMR, p.78-92, 2003.

MERTON, R.C. On the pricing of corporate debt: the risk structure of interest rates. Journal of Finance, p. 449-470, 1974.

MINA, J.; XIAO, J. Y. Return to risk metrics: the evolution of a standard. Risk Metrics Group, 2001.

OLIVEIRA, R. D.; ALMEIDA, C. I. R. de. Alocação de carteiras sujeitas a risco de crédito. Revista Brasileira de Finanças, v. 1, n. 2, p. 301-339, 2003.

SAITO, R.; SHENG, H. H. Determinantes de spread das debêntures no mercado brasileiro. Revista de Administração / Faculdade de Economia, Administração e Contabilidade da Universidade de São Paulo, v.40, n. 2, p. 193-205, 2005.

SAUNDERS, A.; ALLEN, L. Credit risk measurement - new approaches to value at risk and other paradigms. $2^{\text {nd }}$ Edition. John Wiley \& Sons, 2002.

SECURATO, J. R. Uma variação do modelo KMV de crédito para cálculo da probabilidade de default para uma empresa. Universidade de São Paulo - Faculdade de Economia, Administração e Contabilidade - Departamento de Administração, 2003 Disponível em: http://www.ead.fea.usp.br/wpapers

STUDER, G. Value at risk and maximum loss optimization. ETHZ, RiskLab Technical Report, 1995. 


\section{ANEXO I - RISCO DE CRÉDITO AGREGADO PARA UMA CARTEIRA DE DEBENTURES}

Conforme exposto na seção 2, o preço de uma debênture pode ser expresso pela equação (6). Para o cálculo do risco, precisamos estimar o preço de cada debênture para um instante futuro, de forma a simular o impacto da variação de preço sobre o retorno da carteira. Assim, devemos inicialmente expressar o preço em função do valor do ativo da empresa emissora e da volatilidade deste valor para um prazo determinado, no nosso caso, de um ano:

$$
\begin{aligned}
P(1, T) & =\sum_{i=1}^{2 T-1} D\left(1, T_{i}\right)\left[(c / 2) N\left(d_{2}\left(c / 2, T_{i}\right)\right)+V_{1} N\left(-d_{1}\left(c / 2, T_{i}\right)\right) D^{-1}\left(1, T_{i}\right)\right] \\
& +D(1, T)\left[(1+c / 2) N\left(d_{2}(1+c / 2, T)\right)+V_{1} N\left(-d_{1}(1+c / 2, T)\right) D^{-1}(1, T)\right]
\end{aligned}
$$

onde $\left(V_{l}\right)$ é o valor de mercado dos ativos da empresa emissora no horizonte de um ano, $\left(D\left(l, T_{i}\right)\right)$ denota o valor presente em $t=l$ de um bônus "zero-cupom" com vencimento em $\left(T_{i}\right),(c)$ é o cupom, $(T)$ é a maturidade da debênture, $N($.) representa a função normal padrão acumulada. Considerando o vetor $\alpha=\left[\alpha_{1}, \ldots, \alpha_{N}\right]$ como o vetor dos pesos de cada debênture no portfolio de teste, temos que o retorno do portfolio para o prazo de um ano pode ser expresso como:

$$
R T=\sum_{i} \alpha_{i}\left[\frac{P_{i}(1, T)}{P_{i}(0, T)}-1\right]
$$

onde os pesos $\left(\alpha_{\imath}\right)$ e os preços $\left(P_{i}(0, T)\right)$ são conhecidos para cada debênture $(i)$. Considerando que nossos fatores de risco são os log-retornos dos ativos das empresas emissoras das debêntures e que o conjunto destes fatores tem distribuição normal multivariada com matriz de co-variância $(\Sigma)$, para a determinação do risco de crédito do portfolio para um nível de confiança de $99 \%$ teremos que resolver o seguinte problema de otimização:

$$
\begin{aligned}
& P M=\min _{v} R T\left(V_{1}\right) \\
& \text { s.a. } \quad(v-\mu)^{T} \Sigma^{-1}(v-\mu) \leq \chi_{1 \%, N}^{2}
\end{aligned}
$$

onde $v=\ln \left(\frac{V_{1}}{V_{0}}\right)$ e $\left(\chi_{1 \%, N}^{2}\right)$ é o quantil $1 \%$ da distribuição qui-quadrada com $(N)$ graus de liberdade. 
Intuitivamente, o que queremos determinar é a maior perda que a carteira possa vir a sofrer caso haja variações extremas nos valores dos fatores de risco. Conhecendo essa medida, o investidor poderá tomar providências para se proteger deste risco.

Assim, para resolvermos o problema de otimização I.3 devemos inicialmente determinar o lagrangeano:

$$
\ell(v, \lambda)=R T+\lambda\left(\chi_{1 \%}^{2}-(v-\mu)^{T} \Sigma^{-1}(v-\mu)\right)
$$

onde $(\lambda)>0$ é o multiplicador de Kuhn-Tucker da equação. Logo, as condições de primeira ordem para cada debênture (i) são dadas por:

$$
\frac{\partial R T}{\partial v_{i}}=\lambda 2 e^{T} \Sigma^{-1}(v-\mu)
$$

onde $\left(e^{T}\right)$ é o vetor canônico transposto. Utilizando a equação (I.2), podemos escrever:

$$
\frac{\partial R T}{\partial v_{i}}=\frac{\alpha_{i}}{P_{i}(0, T)}\left[\frac{\partial P_{i}(1, T)}{\partial v_{i}}\right]
$$

Pela regra da cadeia, podemos escrever:

$$
\frac{\partial R T}{\partial v_{i}}=\frac{\alpha_{i}}{P_{i}(0, T)} \frac{\partial P_{i}(1, T)}{\partial V_{1, i}} \frac{\partial V_{1, i}}{\partial v_{i}}
$$

Como $P_{i}(1, T)$ é o preço de uma put sobre o valor dos ativos da empresa $\left(V_{i}\right), \frac{\partial P_{i}(1, T)}{\partial V_{i}}$ é o delta da opção, que vale $N\left(-d_{1}\left(V_{1, i}, T\right)\right)$. Além disso:

$$
\frac{\partial V_{1, i}}{\partial v_{i}}=\frac{1}{\frac{\partial v_{i}}{\partial V_{1, i}}}=\frac{1}{\frac{1}{V_{1, i}}}=V_{1, i}
$$

Substituindo tudo na equação (I.7), temos:

$$
\frac{\partial R T}{\partial v_{i}}=\frac{\alpha_{i}}{P_{i}(0, T)} N\left(-d_{1}\left(V_{1, i}, T\right)\right) V_{1, i}
$$

Voltando na equação (I.5), podemos escrever: 


$$
\frac{\alpha_{i}}{P_{i}(0, T)} N\left(-d_{1}\left(V_{1, i}, T\right)\right) V_{1, i}=\lambda 2 e^{T} \Sigma^{-1}(v-\mu)
$$

Da equação (I.10) podemos achar $V_{1, i}=f(\lambda)$ e substituir os valores na restrição do problema para achar a constante $\lambda$. De posse desta constante, basta voltarmos à equação (I.10) para determinar os valores de mercado dos ativos, que serão usados na equação (I.2) para a determinação da medida PM ou risco do portfolio para um nível de confiança de $99 \%$.

\section{ANEXO II - OBTENÇÃO DO PORTFOLIO ÓTIMO}

Nesta subseção, nosso objetivo será otimizar a função PM mexendo na composição inicial da carteira, de forma a obtermos o mínimo risco dado um retorno esperado $(\mu)$ fixo. Para isso, usaremos a abordagem clássica de média-variância proposta por Markowitz em 1952. Essa teoria permite ao investidor alocar os seus recursos entre vários ativos financeiros, buscando um compromisso ótimo entre retorno e risco. Essa teoria é desenvolvida seguindo a hipótese de que os investidores avaliam suas carteiras com base apenas no valor esperado e na variância das taxas de retorno dos ativos que a compõem, escolhendo sempre as carteiras com maior retorno ou menor risco.

Para determinar a alocação ótima no nosso caso, teremos que resolver simultaneamente dois problemas de otimização. O primeiro para o cálculo do risco:

$$
\begin{aligned}
& P M=\min _{v} R T\left(V_{1}\right) \\
& \text { s.a. } \quad(v-\mu)^{T} \Sigma^{-1}(v-\mu) \leq \chi_{1 \%, N}^{2}
\end{aligned}
$$

onde (v) é o vetor de log-retornos dos ativos, $(\Sigma)$ é a matriz de co-variância, $\left(\chi_{1 \%, N}^{2}\right)$ é o quantil $1 \%$ da distribuição qui-quadrada com $(N)$ graus de liberdade e $R T$ é o retorno total da carteira, expresso pela equação (8).

O segundo para a minimização deste risco para um valor fixo de $(\mu)$ :

$$
\begin{gathered}
\min _{\alpha} P M \\
\text { s.t. } \quad \sum_{i} \alpha_{i} r_{i}=\mu \\
\sum_{i} \alpha_{i}=1
\end{gathered}
$$


onde $\left(r_{i}\right)$ são os retornos esperados de cada debênture para o horizonte de tempo de um ano e $(\mu)$ é o retorno esperado do portfolio para o mesmo prazo.

Oliveira e Almeida (2003) oferecem uma solução para dois programas parecidos, a qual será aproveitada neste trabalho. Comecemos, inicialmente, determinando as condições de primeira ordem do problema (P2). O Lagrangeano pode ser expresso da seguinte forma:

$$
\ell\left(\lambda_{\mu}, \lambda_{2}, \alpha_{i}\right)=P M+\lambda_{\mu}\left(\mu-\sum_{i} \alpha_{i} r_{i}\right)+\lambda_{2}\left(1-\sum_{i} \alpha_{i}\right)
$$

onde $\left(\lambda_{\mu}\right)$ e $\left(\lambda_{2}\right)$ são os multiplicadores de Lagrange de (II.1). As condições de primeira ordem do problema são:

$$
\frac{\partial P M}{\partial \alpha_{i}}=\lambda_{\mu} r_{i}+\lambda_{2}
$$

Aplicando o Teorema do Envelope para (Pl), temos:

$$
\frac{\partial P M}{\partial \alpha_{i}}=\left[\frac{P_{i}(1, T)}{P_{i}(0, T)}-1\right]
$$

Igualando (II.2) e (II.3), podemos escrever:

$$
\begin{aligned}
& {\left[\frac{P_{i}(1, T)}{P_{i}(0, T)}-1\right]=\lambda_{\mu} r_{i}+\lambda_{2}} \\
& P_{i}(1, T)=P_{i}(0, T)\left(1+\lambda_{\mu} r_{i}+\lambda_{2}\right) \\
& V_{1, i}=P_{i}(1, T)^{-1}\left\{P_{i}(0, T)\left(1+\lambda_{\mu} r_{i}+\lambda_{2}\right)\right\} \\
& V_{1, i}=f_{i}\left(\lambda_{\mu}, \lambda_{2}\right)
\end{aligned}
$$

Ou seja, na equação (II.7) podemos escrever $\left(V_{l, i}\right)$ em função dos valores dos multiplicadores de Lagrange. Voltemos nossa atenção agora para o problema ( $\mathrm{Pl})$. Na seção anterior, vimos que as condiçôes de primeira ordem do problema são dadas pela equação (I.5), apresentada no anexo I. Precisamos, portanto, calcular as derivadas da função $R T$ com relação aos fatores de risco $v_{i}$. Aplicando a regra da cadeia, podemos escrever: 


$$
\frac{\partial R T}{\partial v_{i}}=\frac{\alpha_{i}}{P_{i}(0, T)} N\left(-d_{1}\left(V_{1, i}, T\right)\right) V_{1, i}
$$

Substituindo (II.7) e (I.5) em (II.8), temos:

$$
\begin{aligned}
& \frac{\alpha_{i}}{P_{i}(0, T)} N\left(-d_{1}\left(f_{i}\left(\lambda_{\mu}, \lambda_{2}\right), T\right)\right) f_{i}\left(\lambda_{\mu}, \lambda_{2}\right)=\lambda 2 e^{T} \Sigma^{-1}(v-\mu) \\
& \alpha_{i}=\frac{\lambda 2 e^{T} \Sigma^{-1}(v-\mu) P_{i}(0, T)}{N\left(-d_{1}\left(f_{i}\left(\lambda_{\mu}, \lambda_{2}\right), T\right)\right) f_{i}\left(\lambda_{\mu}, \lambda_{2}\right)} \\
& \alpha_{i}=\lambda g_{i}\left(\lambda_{\mu}, \lambda_{2}\right)
\end{aligned}
$$

Ou seja, na equação (II.10) podemos escrever $\left(\alpha_{\mathrm{i}}\right)$ em função dos multiplicadores de Lagrange de (P1) e de (P2). Substituindo a equação (II.l1) nas restrições de (P2), temos:

$$
\begin{aligned}
& \lambda \sum_{\mathrm{i}} g_{i}\left(\lambda_{\mu}, \lambda_{2}\right) r_{i}=\mu \\
& \lambda \sum_{i} g_{i}\left(\lambda_{\mu}, \lambda_{2}\right)=1
\end{aligned}
$$

Dividindo as duas equações, temos:

$$
\frac{\sum_{\mathrm{i}} g_{i}\left(\lambda_{\mu}, \lambda_{2}\right) r_{i}}{\sum_{i} g_{i}\left(\lambda_{\mu}, \lambda_{2}\right)}=\mu
$$

Logo, com as equações (II.7) e (II.12) chegamos a um sistema de duas equações e duas incógnitas. Ao resolvê-lo, conseguimos determinar os multiplicadores de Lagrange que, usados nas equações (II.7) e (II.11), nos permitem obter a composição ótima (vetor $\alpha$ ) que minimiza a perda por crédito da carteira. 